\title{
Transhumanismus aus Sicht der Philosophischen Anthropologie Helmuth Plessners ${ }^{2}$
}

\author{
Mit den Worten Unmensch und unmenschlich \\ sollte man sparsam sein.
}

Helmuth Plessner

Zusammenfassung: Der Beitrag vergleicht die extra-, trans- und posthumanistischen Utopien der Menschenverbesserung mit der philosophischen Anthropologie von Helmuth Plessner kritisiert den Transhumanismus anhand der drei anthropologischen Grundgesetze, die Plessner in seinem Opus Magnum „Die Stufen des Organischen und der Mensch“ (1928) formuliert. Es wird gezeigt, dass der Transhumanismus mit Plessners erstem anthropologischen Gesetz übereinstimmt: dem Gesetz der „natürlichen Künstlichkeit“ des Menschen. Es gibt jedoch zwei wichtige Unterschiede, die den Transhumanismus zu einer radikalisierten philosophischen Anthropologie machen. Erstens wollen Transhumanisten im Gegensatz zu Plessner die natürliche Künstlichkeit nicht nur beschreiben, sondern auch aktiv befördern. Zweitens bemühen sich die Transhumanisten, das gegenwärtige Mensch-Sein in eine transhumane oder sogar posthumane Lebensform zu verwandeln. Während Plessner die Möglichkeit von Lebensformen jenseits der exzentrischen Position des Menschen nicht für möglich hält wird mit Bezug auf Bienen, Oktopoden und Craniopagus Zwillingen argumentiert, dass die Natur bereits polyzentrische und polyexzentrische Lebensformen kennt und dass die technische Realisierung dieser Lebensformen mindestens logisch nicht ausgeschlossen ist. Gleichwohl gibt es gute Gründe gibt, sich den transhumanistischen Träumen nicht begeistert zu überlassen. Plessners zweites Grundgesetz das Gesetz der „vermittelten Unmittelbarkeit“ - lehrt, dass die Entwicklung von Techniken weder vorhersehbar noch kontrollierbar ist. Und das dritte Grundgesetz - das Gesetz des „utopischen Standorts“- macht deutlich, dass eine Überwindung der konstitutionellen Heimatlosigkeit des Menschen - wenn dies überhaupt möglich wäre - das Ende der menschlichen Lebensform bedeuten würde. In einem Einzeiler zusammengefasst: Transhumanisten lassen sich mit jemandem

1 Übersetzt von Maren Wehrle, der ich für ihre kritischen Kommentare zu einer früheren Version dieses Textes danke.

Ә OpenAccess. (C) 2021 Olivia Mitscherlich-Schoennherr, published by De Gruyter. (cc))BY-NC-ND This work is licensed under the Creative Commons Attribution 4.0 International License. 
vergleichen, der alle Vorbereitungen für eine wilden Party trifft, zu der er selbst allerdings nicht eingeladen sein wird.

\section{Einleitung}

Der Transhumanismus ist eine seit den 1990er Jahren populär gewordene Bewegung, welche die Möglichkeit und Wünschbarkeit sowohl einer Optimierung und Erweiterung (human enhancement) des Menschen als auch dessen Überwindung, d.h. die Transformation zu einer posthumanen Lebensform, untersucht. Dabei bezieht sich der Transhumanismus vor allem auf die Möglichkeiten des medizinischen Gebrauchs neuerer Informations- und Biotechnologien. Im Gegensatz zur traditionellen Medizin hat eine solche Optimierungsmedizin nicht die Heilung oder Vermeidung von Krankheiten zum Ziel, sondern die Verbesserung des gesamten menschlichen Lebens. Nicht allein die Lebensverlängerung, der Kampf gegen Krankheiten und Zeichen des Alterns - oder gar der ,heilige Gral‘ der Unsterblichkeit - stehen dabei im Fokus, sondern auch die Optimierung und Erweiterung des Körpers und seiner Funktionen, z. B. mit Hilfe von elektronischen Implantaten, sowie die Steigerung des menschlichen Glücks, etwa durch pharmazeutische Mittel oder neuronale Stimulation. Extra-, Trans- und Posthumanisten träumen dabei von der Schaffung neuer Lebensformen, die die menschliche Lebensform jeweils qualitativ verbessern, übersteigen oder gar vollständig hinter sich lassen. ${ }^{2}$

Genauso wie im philosophisch-anthropologischen Paradigma, wie es bei Helmuth Plessner zum Ausdruck kommt, stellt auch im Transhumanismus der Mensch das zentrale Anliegen der Untersuchung dar. Beide Ansätze haben dabei ihre Wurzeln in einem säkular-humanistischen Weltbild und legen Nachdruck auf den Umstand, dass die Natürlichkeit des Menschen immer schon künstlich, d.h. durch Kultur und Technik vermittelt, ist. Wo diese ,natürliche Künstlichkeit' bei Plessner Ausdruck der menschlichen Lebensform ist, richtet sich die menschliche Schöpfungskraft im Zeitalter konvergierender Technologien laut dem Transhumanismus zunehmend auf eine fundamentale Transformation der menschlichen

2 In Bezug auf die Unterscheidung zwischen extra-, trans- und postmenschlichen Lebensformen ist anzumerken, dass dies eher eine schrittweise als eine scharf ausgeprägte Unterscheidung ist. Im weiteren Verlauf dieses Beitrags werde ich den Begriff ,Transhumanismus“ meistens als Überbegriff verwenden, es sei denn, eine der drei genannten Varianten wird ausdrücklich erwähnt. Für eine detailliertere Diskussion des Unterschieds zwischen extra-, trans- und posthumanistischen Ansichten zur menschlichen Verbesserung siehe Hans-Peter Krügers Beitrag in diesem Band. 
Lebensform selbst. Der Transhumanismus kann insofern als eine radikalisierte Form der philosophischen Anthropologie bezeichnet werden. Eine solche radikalisierte Anthropologie will den Menschen als durch natürliche Künstlichkeit gekennzeichnete Lebensform nicht nur beschreiben und verstehen, sondern verfolgt darüber hinaus aktiv die Gestaltung transhumaner Lebensformen, auch wenn dies das Ende der gegenwärtigen menschlichen Lebensform bedeuten würde.

Parallel dazu gibt es noch wichtige inhaltliche Unterschiede zwischen beiden Ansätzen. Während viele Transhumanisten der auf Platon und Descartes zurückgehenden dualistischen Auffassung des Menschen huldigen, nimmt Plessner eine kritische Stellung zu diesem Dualismus ein und verteidigt ein monistisches Menschenbild, in welchem die psychophysische Einheit des Lebens zentral steht. Obwohl Plessner sich nie selbst zum Transhumanismus geäußert hat, lässt sich im Hinblick auf sein Werk die Frage stellen, ob der Glaube an die Möglichkeit einer überlegenen, posthumanen Lebensform nicht naiv zu nennen ist. Naiv deshalb, da er die einfache Tatsache übersieht, dass die Technologie, obschon sie ein Produkt des Menschen ist, durch diesen niemals vollständig beherrscht werden kann. Zudem ist fraglich, ob ein solches Optimieren oder Fortentwickeln des Menschen seiner ,konstitutiven Heimatlosigkeit‘, welche nach Plessner die menschliche als exzentrische Lebensform geradezu auszeichnet, wirklich ein Ende machen kann. Das utopische Streben der Transhumanisten scheint von der unrealistischen Hoffnung getrieben zu sein, irgendwann den ultimativen Zustand der Glückseligkeit erreichen zu können.

\section{Die transhumanistische Utopie}

Ideen und Geschichten, die das Erlangen übermenschlicher Eigenschaften oder das Erreichen von Unsterblichkeit zum Inhalt haben, gibt es schon sehr lange. Wir treffen diese nicht nur in vielen religiösen und literarischen Schriften an, wie z. B. im viertausendjährigen Epos von Gilgamesch, sondern auch in mythischen Vorstellungen über die Quellen des ewigen Lebens oder das Lebenselixier. Friedrich Nietzsches Idee des Übermenschen wird ebenfalls den Inspirationsquellen des gegenwärtigen Transhumanismus zugerechnet (Sorgner 2009).

Die Entwicklung solcher Ideen kann dabei nicht unabhängig von der Entwicklung moderner Wissenschaften und Technologien gesehen werden. Starke Impulse erfuhren diese Vorstellungen etwa durch den Darwinismus und die Genetik, in jüngster Zeit haben vor allem sogenannte konvergierende Technologien (Informationstechnologie, Neurowissenschaften, Nanotechnologie und Robotik) transhumanistischen Auffassungen - die technologische Verbesserung oder gar 
Transzendierung der menschlichen Lebensform betreffend - zu neuem Aufwind verholfen (De Mul 2010, 2014). ${ }^{3}$

Der Begriff ,Transhumanismus' taucht zum ersten Mal sporadisch in den 1940er Jahren auf, aber es war der Biologe Julian Huxley, der 1957 einen einflussreichen Artikel mit diesem Namen publizierte und so zum eigentlichen Gründer der gleichnamigen Strömung wurde (Huxley 1957).

Wie der Name schon sagt, knüpft der Transhumanismus, auch als Humanismus+ bezeichnet, beim Gedankengut des modernen Humanismus an. Genau wie die Humanisten gehen die Transhumanisten davon aus, dass der Mensch als Teil der Natur den Naturgesetzen unterworfen ist, sich jedoch als vernünftiges und moralisches Wesen gegenüber sich selbst und anderen verantworten kann und muss. Dabei schließen sich die Transhumanisten vor allem bei der angelsächsischen Variante des Humanismus an, die sich im Gegensatz zur bildungshumanistisch geprägten kontinentalen Tradition mehr an den empirischen Wissenschaften und der Technologie orientiert. Obwohl dem Transhumanismus eine gewisse säkulare Heilserwartung nicht fremd ist, nimmt er in der Regel eine abweisende Haltung gegenüber traditionell religiösen Vorstellungen sowie der Idee einer transzendenten Wirklichkeit, die sich hinter den Erscheinungen verbirgt, ein. Das +, das den Transhumanismus vom Humanismus unterscheidet, zeigt sich dabei vor allem in der radikalen Weise, in welcher das humanistische Prinzip der menschlichen Entwicklung inhaltlich ausgefüllt und bestimmt wird (Humanity+ 2003).

Oben genannte Merkmale kommen deutlich zum Ausdruck in der Definition von Max More, der zusammen mit Tom Morrow das Extropy Institute gründete:

Transhumanism is a class of philosophies that seek to guide us towards a posthuman condition. Transhumanism shares many elements of humanism, including a respect for reason and science, a commitment to progress, and a valuing of human (or transhuman) existence in this life. [...] Transhumanism differs from humanism in recognizing and anticipating the radical alterations in the nature and possibilities of our lives resulting from various sciences and technologies. (More 1990)

Im Jahre 1983 gründeten die Philosophen Nick Bostrom und David Pearce die World Transhumanist Association (WTA), die später umbenannt wurde in $\mathrm{Hu}$ manity+, eine internationale NGO, welche die Anerkennung des Transhumanismus als legitimes Thema wissenschaftlicher Forschung und politischer Regierung

3 Siehe hierzu auch Oliver Müllers Beitrag in diesem Band über die philosophischen und theologischen Tiefenstrukturen des Transhumanismus, der eine Transformation von einem geisteswissenschaftlichen Selbsterschaffungspostulat zu einem biotechnischen Programm zeigt. 
zum Ziel hat. Diese Organisation definiert Transhumanismus wie folgt (Humanity $+2003)$ :

1. The intellectual and cultural movement that affirms the possibility and desirability of fundamentally improving the human condition through applied reason, especially by developing and making widely available technologies to eliminate aging and to greatly enhance human intellectual, physical, and psychological capacities.

2. The study of the ramifications, promises, and potential dangers of technologies that will enable us to overcome fundamental human limitations, and the related study of the ethical matters involved in developing and using such technologies.

Gemäß der Transhumanisten ist der Mensch die erste Spezies der Evolution, die in der Lage sein wird, seine evolutionären Nachfolger selbst zu schaffen. Neben ihrem Streben nach einer Optimierung des Menschen durch pharmazeutische Mittel, die die Intelligenz des Menschen erhöhen, seine Gefühle regulieren und seinen Genuss wie sein Glück verstärken sollen, hoffen die Transhumanisten darauf, die menschliche Lebensform selbst auf eine qualitativ höhere Stufe erheben zu können. Dies soll mit Hilfe von Nanotechnologien oder verschiedenartigen Biotechnologien, wie dem Klonen, oder der genetische Modifizierung nach der CRISPR-Cas9 Methode, gelingen. Dabei stützen die Transhumanisten ihre Hoffnung ebenfalls auf Neurowissenschaften, Informationstechnologie, künstliche Intelligenzforschung und Robotik, die es möglich machen, den Menschen durch technische Hilfsmittel zu optimieren, indem etwa elektronische Implantate und Gehirn-Computer Schnittstellen (brain-computer interfaces) in die menschliche Lebensform integriert werden.

Hierdurch soll es möglich werden, Menschen und Computer zu einer überlegenen Form von Cyborg zu verschmelzen oder sie mit Hilfe von Computernetzwerken zu einem Superorganismus zusammenzufügen. Einige Transhumanisten, die fürchten, eine solche Entwicklung nicht mehr miterleben zu dürfen, lassen sich nach ihrem Tod einfrieren in der Hoffnung, dass die zukünftige Wissenschaft und Technologie sie wieder zum Leben (in welcher Form auch immer) erwecken können wird.

Einer der radikalsten Transhumanisten ist Hans Moravec, der in seinem Buch Mind Children. The Future of Robot and Human Intelligence (1988) eine nicht weniger als kosmologisch $\mathrm{zu}$ nennende Zukunftsperspektive schildert. Ausgangspunkt seines Ausblicks in die Zukunft ist die Fehlerhaftigkeit und Fragilität 
des menschlichen Daseins. ${ }^{4}$ Unser Körper kann leicht beschädigt oder gar zerstört werden, etwa durch Unterernährung, Krankheit, Strahleneinwirkung, Unfälle oder Unglücke etc. Darüber hinaus ist unsere körperliche Kraft sehr gering und schnell erschöpft sowie unsere durchschnittliche Lebenserwartung mit ca. 80 Jahren äußerst kurz.

Die Lösung, die Moravec für die Behebung dieses mangelhaften Zustandes anbietet, ist das ,downloaden' des menschlichen Geistes in den künstlichen und damit zugleich überlegenen Körper eines Roboters. Hierbei hat er einen Roboter vor Augen, der auf dem Gebiet der Gehirnchirurgie spezialisiert ist. Dieser soll unser Gehirn Schicht für Schicht einscannen und danach eine Computersimulation davon in das mechanische Gehirn eines Roboters kopieren. Moravec setzt dabei voraus, dass der Geist nur ein Nebenprodukt oder Epiphänomen des materiellen Gehirns darstellt. Die Identität des Geistes ist daher nicht im Stoff angesiedelt, aus dem das Gehirn besteht, sondern in der Struktur und den Prozessen, die sich darin abspielen. Ein Indiz hierfür sieht Moravec in der Tatsache, dass im Laufe der Zeit alle Zellen in unserem Körper ausgetauscht werden, jedoch die Strukturen und damit der ,Geist" erhalten bleibt. ${ }^{5}$ Auch Moravec sieht voraus, dass der Mensch sich zukünftig mit verschiedenartigsten Implantaten, Prothesen, zusätzlichen Organen und Sinnen ausstatten bzw. ,upgraden` wird. Hierbei erwähnt er auch das Kombinieren von verschiedenen Lebensformen. Warum sollten wir auch nicht die Intelligenz von Delfinen und Elefanten unserer Intelligenz hinzufügen? Letztendlich wird der Mensch sich nach Moravec dafür entscheiden, als bloße Simulation fort zu leben. Dank der Download Prozedur ist der Mensch

4 Für eine detailliertere Auseinandersetzung mit dieser posthumanistischen Annahme der menschlichen Fehlerhaftigkeit und Fragilität vgl. den Beitrag von Armin Grunwald in diesem Band.

5 Unabhängig davon, ob die von Moravec beschriebene Operation jemals technisch möglich wird, setzt das durch Moravec beschriebene Ergebnis der Operation - die Übertragung des Geistes eines Menschen auf den Körper eines Roboters - eine problematische metaphysische Annahme voraus, nämlich dass die Identität des Geistes völlig unabhängig ist von der Art des Körpers, mit dem er verbunden ist. Die Voraussetzung einer von der materiellen Verwirklichung unabhängigen „pattern identity“ des Geistes ist aber schwer vorstellbar. Wenn mit Plessner und gegen Descartes angenommen wird, dass ein Lebewesen kein zufälliges Konglomerat zweier verschiedener Substanzen (Körper und Geist) ist, sondern eine psychophysische Einheit bildet, die gesetzt ist und sich gleichzeitig und ständig in eine Umwelt setzt, dann ist die Unterscheidung von Körper und Geist gar nicht ontologisch, sondern ausschließlich perspektivisch zu verstehen. Das Hochladen des Geistes in eine Maschine wird dann aus philosophischen Gründen entweder ganz unmöglich oder es wird zu einem nicht identischen Geist führen. Im letzteren Fall würde es den Geist mit völlig neuen Problemen wie psychischer Metallermüdung belasten. Eine weitere Erörterung der metaphysischen Schwierigkeiten des Mind-Uploading leistet Tobias Müller in seinem Beitrag zu diesem Band. 
nun auch in der Lage, backups oder zahlreiche Kopien von sich selbst anzufertigen, die sich dann rasend schnell via Computernetzwerken bewegen und ihren Ort wechseln können, etwa um andere Planeten und Sonnensysteme zu besiedeln:

Our speculation ends in a supercivilisation, the synthesis of all solarsystem life, constantly improving and extending itself, spreading outward from the sun, converting nonlife into mind. Just possibly there other such bubbles expanding from elsewhere. What happens if we meet one? A negotiated merger is a possibility, requiring only a translation scheme between the memory representations. This process, possibly occurring elsewhere, might convert the entire universe into an extended thinking entity, a prelude to even greater things. (Moravec 1988, 116)

\section{Eine Plessnersche Perspektive auf den Transhumanismus}

Viele Vorstellungen der Transhumanisten erscheinen auf den ersten Blick als Science-Fiction bzw. als hypermodernistische Orgie von Beherrschbarkeitsfantasien. Jedoch wäre es unklug, den Transhumanismus und seine Ideen nicht ernst zu nehmen. Viele der Technologien, auf die sich die Transhumanisten berufen, sind immerhin schon Realität geworden oder werden in experimentellen Laborumgebungen getestet. In seiner kompromisslosen Radikalität macht der Transhumanismus diejenigen Motive explizit, die die gegenwärtige Wissenschaft und Technologie auf eine oft unausgesprochene Weise anzutreiben scheinen.

Die Motivation des Transhumanismus kann aus Plessners Perspektive vom ersten seiner drei anthropologischen Grundgesetze formuliert werden, die er im letzten Kapitel der „Stufen des Organischen und der Mensch“ erörtert. Seine ,natürliche Künstlichkeit‘ hat demnach den Menschen seit jeher zu dem Versuch angetrieben, der ,konstitutiven Heimatlosigkeit', die seine exzentrische Lebensform $^{6}$ kennzeichnet, mit der Hilfe von technischen Hilfsmitteln zu entfliehen:

6 Exzentrisch bedeutet, dass der Mensch nicht nur ein Körper ist (wie die Pflanze) und darüber hinaus seinen Körper hat (wie das Tier), sondern im gewissen Sinn auch außerhalb seines Körpers ist, seinen Körper und Leib sozusagen von außen betrachten kann: „Positional liegt ein Dreifaches vor: das Lebendige ist Körper, im Körper (als Innenleben oder Seele) und außer dem Körper als Blickpunkt, von dem aus es beides ist“ (Plessner 2003a,, 365). Oder aus der psychischen Perspektive gesehen: „Er lebt und erlebt nicht nur, sondern er erlebt sein Erleben“ (ebd., 364). Dies bedeutet, dass der Mensch nicht vollständig mit sich selbst zusammenfällt, weshalb Plessner ihn konstitutiv heimatlos nennt. 
Exzentrische Lebensform und Ergänzungsbedürftigkeit bilden ein und denselben Tatbestand. Bedürftigkeit darf hier nicht in einem subjektiven Sinne und psychologisch aufgefaßt werden. Sie ist allen Bedürfnissen, jedem Drang, jedem Trieb, jeder Tendenz, jedem Willen des Menschen vorgegeben. In dieser Bedürftigkeit oder Nacktheit liegt das Movens für alle spezifisch menschliche, d.h. auf Irreales gerichtete und mit künstlichen Mitteln arbeitende Tätigkeit, der letzte Grund für das Werkzeug und dasjenige, dem es dient: die Kultur. (Plessner 2003a, 385)

Das Bestreben des Transhumanismus nach Optimierung unserer aktuellen Lebensform reicht in eine lange Tradition der Technikgeschichte zurück. Laut Plessner zeichnet sich die Menschheit weiterhin durch ihre unablässige Geschichtlichkeit aus: „Durch seine Expressivität ist er also ein Wesen, das selbst bei kontinuierlich sich erhaltender Intention nach immer anderer Verwirklichung drängt und so eine Geschichte hinter sich zurückläßt“ (Plessner 2003a, 416). Die Träume und Praktiken menschlicher Optimierung sind daher unauflösbar mit der menschlichen Lebensform verbunden. Aus dieser Perspektive lässt sich eine kontinuierliche Entwicklung feststellen, vom Herstellen und Gebrauchen von Werkzeugen, Kleidung, Feuer, Waffen und der Schrift bis hin zu der Entwicklung von Exoskeletten, Gehirn-Computer Schnittstellen und genetischer Modifizierung.

Zugleich ist Plessner jedoch besonders zurückhaltend, wenn es um die Möglichkeit einer Entwicklung geht, die über die exzentrische Lebensform hinausweist:

$\mathrm{Zu}$ immer neuen Akten der Reflexion auf sich selber, zu einem regressus ad infinitum des Selbstbewußtseins ist auf dieser äußersten Stufe des Lebens der Grund gelegt und damit die Spaltung in Außenfeld, Innenfeld und Bewußtsein vollzogen.

Man begreift, warum die tierische Natur auf dieser höchsten Positionsstufe erhalten bleiben muß. Die geschlossene Form der Organisation wird nur bis zum Äußersten durchgeführt. Zeigt doch das lebendige Ding in seinen positionalen Momenten keinen Punkt, von dem aus eine Steigerung erzielt werden könnte, außer durch Verwirklichung der Möglichkeit, das reflexive Gesamtsystem des tierischen Körpers nach dem Prinzip der Reflexivität zu organisieren und das, was auf der Tierstufe das Leben nur ausmacht, noch in Beziehung zum Lebewesen $\mathrm{zu}$ setzen. Eine weitere Steigerung darüber hinaus ist unmöglich, denn das lebendige Ding ist jetzt wirklich hinter sich gekommen. (Plessner 2003a, 363; Kursivierung JdM)

Plessner scheint hier einer geschlossenen Hegelschen Dialektik zu folgen. Mit Blick auf die seit vier Millionen Jahren andauernde Evolution des Lebens scheint mir das ziemlich naiv zu sein. Umso mehr, da Plessner in den Stufen betont, dass seine Rekonstruktion der aufeinanderfolgenden Stufen der lebendigen Formen nicht im eigentlichen Sinne apriorisch ist, ,als wolle sie aus reinen Begriffen unter Beziehung von Axiomen ein deduktives System entwickeln, sondern nur kraft ihrer regressiven Methode, zu einem Faktum seine inneren ermöglichenden Bedingungen zu finden“ (Plessner 2003a, 29-30). 
Empirische Forschung kann immer neue Fakten liefern, die eine Überarbeitung der von Plessner ausgezeichneten Stufen erfordern. In jedem Fall lässt die jüngste biologische Forschung Zweifel aufkommen, ob das Stufen-Modell von Plessner erschöpfend ist. Die biologische Forschung wirft zum Beispiel die Frage auf, ob die zentrische Position, die Plessner Tieren zuschreibt, die einzig mögliche ist. So kann man bezweifeln, ob es bei Honigbienen richtig ist, einzelne Bienen als Organismen zu betrachten. Angesichts der Verteilung der Lebensfunktionen auf Königin, Arbeiterinnen und Drohnen wird behauptet, dass nicht die einzelnen Bienen die Organismen sind, sondern der Schwarm insgesamt (Hölldobler/Wilson 2009). In diesem Fall würden wir eher von einer polyzentrischen Lebensform sprechen, bei der die Lebensfunktionen auf mehrere ,Dividuen“ verteilt sind.

Des Weiteren werfen Forschungen zu Oktopoden beispielsweise die Frage auf, ob nicht auch die exzentrische Position, die Plessner dem Mensch zuschreibt, andere Formen annehmen kann. Peter Godfrey-Smith stellt in seinem Buch Other Minds. The Octopus, the Sea, and the Deep Origins of Consciousness (2016) dar, dass es sich hier um eine intelligente Lebensform handelt, die sich radikal von den Wirbeltieren unterscheidet, zu denen Tiere, einschließlich Menschen, gehören:

Cephalopods are an island of mental complexity in the sea of invertebrate animals. Because our most recent common ancestor was so simple and lies so far back [ca. 750 million years $\mathrm{JdM}$, cephalopods are an independent experiment in the evolution of large brains and complex behavior. If we can make contact with cephalopods as sentient beings, it is not because of a shared history, not because of kinship, but because evolution built minds twice over. This is probably the closest we will come to meeting an intelligent alien. (Godfrey-Smith 2016, 9).

Die mindestens 500 Millionen Neuronen des Oktopodes befinden sich nicht nur im Kopf, sondern sind im ganzen Körper verteilt. Die Unterscheidung zwischen Gehirn (Zentrum) und Körper scheint hier überhaupt nicht zuzutreffen. Da sich zwei Drittel der Neuronen in den acht Tentakeln befinden, bilden diese jeweils ein autonomes Erlebniszentrum und können jeweils das Verhalten des Oktopodes steuern. Man könnte hier von einer polyzentrischen Positionalität sprechen. Experimente zeigen, dass Tintenfische ein sehr intelligentes und exploratives Verhalten zeigen und zum Beispiel verschiedene menschliche Individuen unterscheiden können. Es ist auch nicht undenkbar, dass sie wie Menschenaffen, Delfine und Elefanten ein gewisses Maß an Selbstbewusstsein haben. Es ist bereits unmöglich, sich wirklich vorzustellen, wie es ist, ein Säugetier wie die Fledermaus zu sein, obwohl wir eine zentrische Positionalität teilen (Nagel, 1974). Sich vorzustellen, was es bedeutet, ein poly(ex)zentrisches Tier wie ein Oktopode zu sein, scheint - obwohl wir vielleicht Selbstbewusstsein teilen - eine Super- 
lative des Unmöglichen zu sein. Der Bauplan des Oktopodes erfordert jedoch zumindest die Anerkennung des vorläufigen Charakters der von Plessner beschriebenen Stufen.

Damit ist natürlich nicht gesagt, dass sich ausgehend vom gegenwärtigen Menschen trans- oder posthumane Lebensformen entwickeln werden, aber ebenso wenig, dass dies kategorisch ausgeschlossen werden kann. Wenn es nach den Transhumanisten ginge, müssten wir sogar engagiert danach streben, das lebendige Ding wirklich über sich hinaus kommen zu lassen.

Auch in der Forschungsliteratur zu Plessner sind bereits Versuche unternommen worden, um - mit Plessner gegen Plessner - über neue Positionalitätsstufen zu spekulieren, die jenseits der exzentrischen Positionalität zu verorten sind. Anders als bei Transhumanisten sind solche Spekulationen hier nicht mit einem „technologischen Imperativ“ verbunden, sondern mit der offenen Frage, ob wir solche neuen Stufen verfolgen oder verhindern sollen.

Insbesondere die jüngsten Entwicklungen bei den sogenannten konvergierenden Technologien scheinen eine Herausforderung für die menschliche Lebensform darzustellen, wie sie seit etwa zweihunderttausend Jahren besteht. Die vielfältigen Möglichkeiten mit Hilfe psychotropischer Drogen, tiefer Hirn-Stimulation sowie intervenierender Genetik in das menschliche Bewusstsein einzugreifen, um etwa Einfluss auf unsere Stimmungen, Konzentrationsmöglichkeiten oder gar auf bestimmte Charakterzüge auszuüben, geben zum Beispiel Anlass zu Spekulationen über eine meta-exzentrische Positionalität (Verbeek 2014, 453).

Auch die Entwicklung und Anwendung von Technologien der Telepräsenz, wie z. B. eines Teleroboters, von dessen Gliedern und Sinnen der Benutzer jeweils Gebrauch machen kann, und die damit verbundene Multiplikation des Erfahrungszentrums zeigen etwa die Möglichkeit einer artifiziellen Realisierung der polyzentrischen Exzentrizität in Menschen auf (De Mul 2003).

Die Tatsache, dass es Fälle von lebenden siamesischen (craniopagen) Zwillingen gibt, wirft sogar die Frage auf, ob sich die menschliche Evolution auch in Richtung einer Polyexzentrizität bewegen könnte. ${ }^{7}$ Craniopagus-Zwillinge wie die in 2006 geborene Tatiana und Krista teilen einen Schädel und Blutkreislauf. Obwohl jede von beiden ein vollständiges Gehirn und ein eigenständiges Bewusstsein mit je einer eigenen Ich-Perspektive hat, gibt es auch eine ungewöhnliche Verbindung zwischen dem Thalamus der beiden Mädchen. Es handelt

7 Die folgenden vier Absätze stammen teilweise aus meinem Artikel „Polyzentrizität und Poly (ex) zentrizität: neue Stufen der Positionalität? Zu Telerobotern, craniopagen Zwillingen und globalen Gehirnen“ (De Mul 2018a). 
sich dabei um den Teil des Gehirns, der bei der Regulation von Bewusstsein und der Weiterleitung sensorischer Signale eine wichtige Rolle spielt.

Die Thalamus-Brücke zwischen ihren Gehirnen verursacht sehr seltsame Phänomene. In einer CBC TV Dokumentation berichtet ein Arzt zum Beispiel, dass, wenn eines der beiden Kinder gekitzelt wird, das andere zu lachen anfängt und steckt man einen Schnuller in den Mund des einen Kindes, so hört das andere auf zu weinen (Pyke 2014). Die Dokumentation zeigt, wie beim Schauen eines Fernsehfilmes durch das eine Mädchen das andere den Film tatsächlich mitschaute, durch die Augen ihrer Schwester. Die visuelle Information geht dabei von einem Augenpaar zu beiden Zwillingen. Die Ärzte vermuten, dass der visuelle Input von der Retina des einen Mädchens entlang ihrer optischen Nerven geht und dann in zwei Teile aufgespalten wird. Einer nimmt den üblichen Weg zu ihrem visuellen Kortex, der andere passiert die Thalamus-Brücke zum Thalamus der Schwester und gelangt von dort zum visuellen Kortex. Diese Beispiele zeigen, dass die Mädchen, genau wie im Falle telerobotischer Assemblagen, polyzentrische Erfahrungen machen (indem sie in der Lage sind, die Gedanken des jeweils anderen Geschwisterteils zu denken, da ihre Gehirne den sensorischen Apparat des anderen nutzen). Ähnlich wie Bienen können diese Zwillinge als polyzentrisch bezeichnet werden. Wie die Bienen verfügen sie über ein „Schwarmgehirn“.

Doch sie scheinen darüber hinaus in der Lage zu sein, wechselseitig ihre Gedanken zu lesen, das heißt, sie sind sich introspektiv des mentalen Status bewusst, in dem sich das Bewusstsein der Schwester befindet. Sie sind, mit anderen Worten, in der Lage, im Wortsinn die Perspektive der anderen zu übernehmen. Die CBC TV Dokumentation macht deutlich, dass obwohl sich die Kinder voll bewusst sind, verschiedene Personen zu sein und sie zwischen ihren IchPerspektiven unterscheiden können, sie manchmal die andere für sich selbst oder sich selbst für die andere nehmen.

Philosophisch ist dies ein sehr interessanter Aspekt. Wittgenstein folgend würden die meisten Philosophen argumentieren, dass auf Introspektion basierte Selbst-Zuschreibungen mentaler Zustände gegen Fehler aufgrund von FehlIdentifikation immun sind. Dies gilt, wenn jemand über Zahnschmerzen klagt, so argumentiert Wittgenstein in The Blue Book: „To ask, are you sure it is you who have pains?' would be nonsensical“ (Wittgenstein 1969, 67). Tatsächlich scheint es absurd anzunehmen, jemand könnte der Meinung sein, er fühle die Zahnschmerzen eines anderen. Es ist allgemein akzeptiert, dass eine solche FehlIdentifikation eine logische und metaphysische Unmöglichkeit darstellt. Gleichwohl zeigt der Fall von Tatiana und Krista Hogan - wie Peter Langland-Hassan in seinem Aufsatz „Introspective misidentification“ argumentiert (Langland-Hassan 2015, S. 1754) - die Möglichkeit genau einer solchen Fehlidentifikation. Indem die Mädchen tatsächlich den Schmerz der anderen über die Thalamus-Brücke fühlen, 
ist es weder eine logische noch eine metaphysische Unmöglichkeit, dass sie versehentlich den Schmerz der Schwester für ihren eigenen halten. In diesem Sinne sind die Schwestern nicht nur polyzentrisch, sondern auch poly-exzentrisch zu nennen.

Transhumanisten träumen von der Möglichkeit der Entwicklung von künstlichen Thalamus-Brücken, welche menschliche Individuen verbinden, um somit neue Ebenen menschlicher Kommunikation und koordinierter Handlung zu erreichen. Diese künstlichen Thalamus-Brücken würden nicht wie die MenschMaschine-Interfaces funktionieren, die bereits existieren (wie die Experimente mit Patienten, die unter Rückenmarksverletzungen leiden und mit Hilfe ähnlicher Mensch-Maschine-Interfaces in der Lage sind, einen Roboterarm oder Computer mit ihren Gedanken zu steuern), sondern als Mensch-Mensch-Interfaces, welche es den verbundenen Personen ermöglichen, buchstäblich Gedanken, Willen und Gefühle zu teilen. Der evolutionäre Erfolg des Homo sapiens gegenüber anderen Hominiden wird häufig mit seinen Fähigkeiten erklärt, die Intention anderer zu verstehen und zu teilen (kollektive Intentionalität), andere extensiv $\mathrm{zu}$ imitieren, sich gegenseitig etwas beizubringen und miteinander $\mathrm{zu}$ kommunizieren, um Gedanken oder Handlungen weiterzugeben und zu teilen (Tomasello 2016). Die kontinuierlich wachsende Kooperation zwischen Individuen wurde dabei durch die Entwicklung immer effizienterer Kommunikationsmedien ermöglicht, von der gesprochenen Sprache über die Schrift und Druckerpresse bis hin zur gegenwärtigen Telekommunikation sowie Computernetzwerken - und diese schließlich erweitert durch Algorithmen, Datamining und Ähnlichem. Kollektive Projekte wie Wikipedia werden häufig als das Ergebnis von menschlicher Schwarmintelligenz dargestellt und als die Entstehung einer kollektiven Intelligenz. Vielleicht sind es Vorstufen zu posthumanen Lebensformen, die uns an ,den Borg، aus der Serie Star Trek denken lassen, einen Superorganismus, der alle anderen Lebensformen in sich assimiliert (vgl. De Mul 2018a und 2018b).

Nehmen wir einmal an, dass die natürliche Künstlichkeit des Menschen letztendlich zu post-exzentrischen Positionalitätsstufen führt, dann bleibt natürlich die Frage, ob die Form dieser Stufen dieselbe sein wird, die der Mensch gegenwärtig vor Augen hat bzw. sich vorzustellen in der Lage ist. Das scheint mir nicht weniger unmöglich, als uns vorzustellen, wie es ist, ein Oktopode zu sein. Und es gibt noch ein weiteres ernstes Problem für Transhumanisten. Obwohl der Mensch Schöpfer der Technologie ist, heißt dies nicht, dass er diese auch beherrscht. Dies ist eine der Implikationen des zweiten anthropologischen Grundgesetzes, der ,vermittelten Unmittelbarkeit‘, die zur Folge hat, dass die Schöpfungen des Menschen dessen eigene Intentionalität stets transzendieren: 


\begin{abstract}
Glaubt man also, daß die Dinge unseres Umgangs und Gebrauchs den vollen Sinn, ihr ganzes Dasein erst aus der Hand des Konstrukteurs empfangen und allein in dieser Relativität auf das Umgehen mit ihnen wirklich sind, so sieht man nur die halbe Wahrheit. Denn ebenso wesentlich ist für die technischen Hilfsmittel (und darüber hinaus für alle Werke und Satzung aus menschlicher Schöpferkraft) ihr inneres Gewicht, ihre Objektivität, die als dasjenige an ihnen erscheint, was nur gefunden und entdeckt, nicht gemacht werden konnte. (Plessner 2003a, 396-397)
\end{abstract}

Transhumanisten, die mit Nietzsche die Schöpfung einer posthumanen Lebensform anstreben, die per definitionem in ihren Vermögen und Erfahrungen weit über sich selbst hinaus geht, lassen sich dabei vergleichen mit jemandem, der alle Vorbereitungen für eine wilde Party trifft, zu der er selbst allerdings nicht eingeladen sein wird.

Die Frage ist freilich, ob wir darüber betrübt sein müssen. Es gibt nämlich keine einzige Garantie dafür, dass eine trans- oder posthumane Lebensform uns ein glücklicheres Dasein verschaffen kann. Transhumanisten werden - so würde Plessner im Hinblick auf das letzte seiner drei anthropologischen Grundgesetze, das Gesetz des ,utopischen Standort', argumentieren - so wie alle Menschen angetrieben durch die utopische Hoffnung, dass sich ihre konstitutive Heimatlosigkeit irgendwann erfolgreich überwinden lässt (Coenen 2017). Obwohl solche religiösen, politischen oder technologischen Träume in Momenten der Trauer und Hoffnungslosigkeit Trost bieten können, haben sie wenig mit der Realität gemein. Insofern die Heimatlosigkeit für den Menschen konstitutiv ist, würde ihre Überwindung nicht nur das Ende der menschlichen Lebensform bedeuten, sondern damit auch das Ende unserer menschlichen, allzu menschlichen Träume selbst.

Und doch ist ein solches Ende des Menschen aus Plessners Perspektive nicht undenkbar. Mit der in der Exzentrizität angelegten natürlichen Künstlichkeit des Menschen ist nämlich auch die Möglichkeit der Unmenschlichkeit gegeben:

Eine prometheische Kultur hat kein Maß und kennt kein Tremendum. Sie steht unter dem Gesetz der Grenzenlosigkeit des Könnens und des Siegens über alle Widerstände, ein Prinzip, das seine faustische Anfänge, wenn überhaupt, längst hinter sich gelassen hat. [...] Unmenschlichkeit ist an keine Epoche gebunden und an keine geschichtliche Größe, sondern eine mit dem Menschen gegeben Möglichkeit, sich und seinesgleichen zu negieren. (Plessner 2003b, 334).

\title{
Literatur
}

Coenen, Christopher (2007): Utopian aspects of the debate on converging technologies (PDF), in: Banse, Gerhard et al.: Assessing Societal Implications of Converging Technological Development, Berlin, 141-172. 
Godfrey-Smith, Peter (2018). Other Minds. The Octopus, the Sea, and the Deep Origins of Consciousness, New York.

Grunwald, Armin: Technische Zukunft des Menschen? Eschatologische Erzählungen zur Digitalisierung und ihre Kritik, im vorliegenden Band.

Hölldobler, Bert/Edward O. Wilson (2009). The Superorganism. The Beauty, Elegance, and Strangeness of Insect Societies, New York.

Humanity+ (2003): What is transhumanism?, unter: https://whatistranshumanism.org/, abgerufen am 8. Februar 2021.

Huxley, Julian (1957). Transhumanism, in: New Bottles for New Wine, London, 13-17.

Krüger, Hans-Peter: Für die Integration künstlicher neuronaler Netzwerke in die personale Lebensform. Eine philosophisch-anthropologische Kritik an der posthumanistischen Superintelligenz, im vorliegenden Band.

Langland-Hassan, Peter (2015). Introspective Misidentification, in: Philosophical Studies 172, 1737-175.

Moravec, Hans P. (1988): Mind Children. The Future of Robot and Human Intelligence, Cambridge, Mass.

More, Max (1990): Transhumanism - Towards a futurist philosophy, unter: https://en.wikipedia.org/wiki/Transhumanism, abgerufen am 8 Februar 2021.

Müller, Oliver: Von der Selbstüberschreitung zur Selbstersetzung. Zu einigen anthropologischen Tiefenstrukturen des Transhumanismus, im vorliegenden Band.

Müller, Tobias: Die transhumanistische Utopie des Mind-Uploading und die Grenzen der technischen Manipulation menschlicher Subjektivität, im vorliegenden Band.

Mul, Jos de (2003): Digitally mediated (dis)embodiment. Plessner's concept of excentric positionality explained for Cyborgs, in: Information, Communication \& Society 6/2, 247265.

Mul, Jos de (2010): Transhumanism - The convergence of evolution, humanism and information technology, in: Mul, Jos de: Cyberspace Odyssey. Towards a Virtual Ontology and Anthropology, Newcastle upon Tyne, 243-262.

Mul, Jos de (2014): Philosophical anthropology 2.0, in: Mul, Jos de (Hg.): Plessner's Philosophical Anthropology. Perspectives and Prospects, Amsterdam/Chicago, 457-475.

Mul, Jos de (2018a): Polyzentrizität und Poly(ex)zentrizität: neue Stufen der Positionalität? Zu Telerobotern, craniopagen Zwillingen und globalen Gehirnen, in: Henkel, Anna/ Lindemann, Gesa (Hg.): Mensch und Welt im Zeichen der Digitalisierung, Baden-Baden, $185-208$.

Mul, Jos de (2018b). Encyclopedias, hive minds and global brains. A cognitive evolutionary account of Wikipedia, in: Romele, Alberto/Terrone, Enrico (Hg.): Towards a Philosophy of Digital Media, Basingstoke/New York, 103-119.

Nagel, Thomas (1974). What is It like to be a bat?, in: The Philosophical Review 83/4, 435-450.

Plessner, Helmuth (2003a): Die Stufen des Organischen und der Mensch. Einleitung in die philosophische Anthropologie [1928], in: Gesammelte Schriften, Band IV, Frankfurt am Main.

Plessner, Helmuth (2003b): Das Problem der Unmenschlichkeit [1967], in: Conditio Humana. Gesammelte Schriften, Band VIII, Frankfurt am Main.

Pyke, Judith (2014). Twin Life: Sharing Mind and Body, Documentary CBC Television, veröffentlicht am 02.10.2014. 
Sorgner, Stefan Lorenz (2009): Nietzsche, the overhuman, and transhumanism, in: Journal of Evolution and Technology. 20 (1), 29-42.

Tomasello, Michael (2016). A Natural History of Human Morality, Harvard.

Verbeek, Peter-Paul (2014): Plessner and technology. Philosophical anthropology meets the posthuman, in: Mul, Jos de (Hg.): Plessner's Philosophical Anthropology. Perspectives and Prospects, Amsterdam/Chicago, 443-456.

Wittgenstein, Ludwig (19692). Preliminary studies for the „Philosophical investigations“, generally known as the Blue and Brown books, New York. 
